\title{
ENFERMEDAD PULMONAR OBSTRUCTIVA CRÓNICA Y COCCIÓN DE ALIMENTOS CON BIOMASA: UN PROBLEMA TRANSDISCIPLINAR.
}

\author{
CHRONIC OBSTRUCTIVE PULMONARY DISEASE AND BIOMASS FOOD COOKING: A TRANSDISCIPLINARY \\ PROBLEM.
}

Ramírez Roldán Sebastián*.

*Fundación Universitaria Juan de Castellanos. Tunja - Boyacá | Colombia.

\section{RESUMEN}

Introducción: La enfermedad pulmonar obstructiva crónica es una de las principales causas de mortalidad a nivel mundial, clásicamente asociada al tabaquismo, los datos indican su importante relación con la inhalación de productos de la combustión de material de biomasa, en el contexto de poblaciones rurales con limitación en el acceso a energías limpias la preparación de alimentos se convierte en factor de riesgo para el padecimiento de esta patología. Objetivo: Proponer la enfermedad pulmonar obstructiva crónica asociada a humo de biomasa en contexto rural como un problema de alta complejidad que requiere de un abordaje transdisciplinar que permita la generación de soluciones ajustadas a las necesidades de esta población. Conclusiones: El enfoque transdisciplinar ofrece una alternativa válida e inexplorada para el estudio de la enfermedad pulmonar obstructiva crónica asociada al humo de biomasa.

Palabras Clave: EPOC, Cocción de alimentos, Transdisciplinar.

\section{ABSTRACT}

Introduction: Chronic obstructive pulmonary disease is one of the main causes of mortality worldwide, classically associated with smoking, the data indicate its important relationship with the inhalation of biomass combustion products, in the context of rural populations with limited access to clean energy, cooking becomes a risk factor for non-communicable diseases such as chronic obstructive pulmonary disease. Objective: To propose chronic obstructive pulmonary disease due to biomass smoke in the rural context as a highly complex problem which requires a transdisciplinary approach that allows generating solutions tailored to the needs of this population. Conclusions: The transdisciplinary approach offers a valid and unexplored alternative to chronic obstructive pulmonary disease research.

Key words: COPD, Food cooking, Transdisciplinary.

Correspondencia: Sebastián Ramírez Roldán sramirezr@jdc.edu.co,

Recibido: 03 de septiembre 2021, aceptado: 22 de noviembre 2021

(C) Autor2022

\section{(c) (i)}

Citation: Ramírez Roldán S. (2022) Enfermedad Pulmonar Obstructiva crónica y cocción de alimentos con biomasa: Un problema transdisciplinar. Revista Salud Pública y Nutrición, 21 (1), 36-42. 


\section{Introducción}

Desde el principio de la vida, comer y nutrirse han sido fundamentales para el desarrollo integral de la especie humana, siendo absolutamente necesarias para su subsistencia. Sin embargo, en lo que respecta a la alimentación humana engloba aspectos más allá de lo biológico, se debe entender la alimentación como un proceso que no se limita a la mera ingesta de alimentos sino que articula una cadena de procesos que involucran la siembra, la cosecha, la distribución, la cocción y la ingesta (Figura 1.); y por tanto, a través de ella el ser humano estimula sus sentidos, se identifica, se expresa, se comunica y socializa con sus pares, o definida por Casanueva como el: "conjunto de procesos biológicos, psicológicos y sociológicos relacionados con la ingestión de alimentos, mediante el cual el organismo obtiene del medio los nutrientes que necesita, así como satisfacciones intelectuales, emocionales, estéticas y socioculturales que son indispensables para la vida humana plena" (Casanueva et al, 2008)

Figura 1. Procesos involucrados en la alimentación.

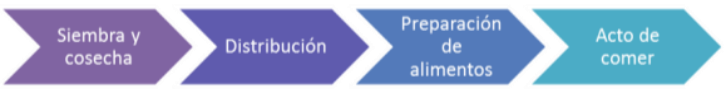

Fuente: Elaboración propia

Comprender la enfermedad pulmonar asociada al humo de biomasa como un problema ligado directamente a la alimentación entorno a la preparación de los alimentos, permite una visión más amplia del problema y a su vez ofrece la oportunidad de ser abordado desde un enfoque transdisciplinar que favorezca el entendimiento de este fenómeno de manera integral, sin limitarla a la definición simplista de salud como "ausencia de enfermedad", aportando a la generación de nuevo conocimiento.

De manera tradicional, la EPOC se ha asociado principalmente al tabaquismo, lo cual, en ocasiones minimiza el impacto de esta patología en personas expuestas de manera crónica a humo producto de combustión de biomasa, en especial mujeres y niñas que desde temprana edad se encuentran desempeñando tareas domésticas como la cocina durante varias horas al día enmarcado en un contexto cultural propio de las comunidades rurales.
De allí la necesidad e insistencia sobre un abordaje transdisciplinar de la problemática, de modo que se permita, desde la ciencia, aportar soluciones que vayan más allá del tratamiento de una enfermedad o posiciones prohibicionistas que trasgredan la cultura material e inmaterial de las comunidades rurales, y a su vez, conlleven al empoderamiento y dignidad de la mujer campesina.

La enfermedad pulmonar obstructiva crónica (EPOC) es la tercera causa de muerte a nivel mundial con más de 3 millones de decesos por año, la cual afecta aproximadamente al $10 \%$ de la población mundial (Celli y Wedzicha, 2019) encontrando una prevalencia mayor en adultos mayores de 40 años. Se caracteriza por alteración de la dinámica de flujo de aire pulmonar, difícilmente reversible, secundario a procesos de inflamación crónica debida a alteración de la arquitectura broncoalveolar producida por exposición a gases o material particulado.

El tabaquismo está catalogado como la principal causa de esta patología, con una prevalencia en adultos para el año 2018 según datos del Banco Mundial del 23.82\% (Banco Mundial, 2018), de los cuales, según datos de la Organización Mundial de la Salud (OMS) el 80\% reside en países de ingresos medios o bajos (OMS, 2021), sin embargo, aproximadamente un tercio de los pacientes diagnosticados con EPOC no han tenido exposición a humo de tabaco, por tanto, se reconocen otras causas como lo es la exposición crónica a biomasa tipo leña, carbón o estiércol, que son usados como biocombustibles en países pobres para la cocción de alimentos o calefacción (Agustí y Hogg, 2019).

El humo producto de la combustión de biomasa está compuesto principalmente por monóxido de carbono (CO), óxido nítrico y sulfúrico, benceno, benzopireno, radicales libres, aldehídos y de partículas de materia respirables (PM), estas últimas son clasificadas según su diámetro aerodinámico, encontrando las denominadas PM10 que corresponden a partículas "gruesas" al tener diámetro de $10 \mu \mathrm{m}$ y las PM2.5 o partículas "finas" de $2.5 \mu \mathrm{m}$ de diámetro, siendo las PM2.5 de particular importancia dada su capacidad de daño a nivel distal de las vías aéreas incluidos los alveolos pulmonares (Bălă, 2021) (Rosário Filho et al, 2021). 
Por ello, la Organización Mundial de la Salud reconociendo el potencial lesivo de éstas partículas emitió en 2006 el documento titulado "WHO Air quality guidelines for particulate matter, ozone, nitrogen dioxide and sulfur dioxide "(WHO, 2006) en el cual se establecen los valores estándar límite de exposición a material particulado sobre los cuales existe el menor riesgo de mortalidad por cáncer pulmonar o enfermedad cardiopulmonar (Tabla 1.). Sin embargo, existen estudios donde se ha calculado la presencia de material particulado en hogares que usan combustión de biomasa para cocinar se han encontrado niveles de material particulado que francamente exceden los valores guía de la OMS representando un riesgo importante para la salud (Zambrano, 2017).

Tabla 1. Valores guía para PM10 y PM2.5 según OMS.

\begin{tabular}{|c|c|}
\hline $\begin{array}{c}\mathrm{PM}_{10}\left(\mu \mathrm{g} / \mathrm{m}^{3}\right)- \\
\text { Anual }\end{array}$ & $\mathrm{PM}_{2.5}\left(\mu \mathrm{g} / \mathrm{m}^{3}\right)-$ Anual \\
\hline 20 & 10 \\
\hline $\mathrm{PM}_{10}\left(\mu \mathrm{g} / \mathrm{m}^{3}\right)-24 \mathrm{~h}$ & $\mathrm{PM}_{2.5}\left(\mu \mathrm{g} / \mathrm{m}^{3}\right)-24 \mathrm{~h}$ \\
\hline 50 & 25 \\
\hline
\end{tabular}

Fuente: (2006). Air quality guidelines: global update 2005: particulate matter, ozone, nitrogen dioxide, and sulfur dioxide. WHO.

Se calcula que aproximadamente el $50 \%$ de las muertes por esta patología en países en vías de desarrollo son atribuibles a la exposición a biomasa, dentro de las cuales el $75 \%$ corresponde a mujeres debido a rasgos culturales en donde ellas tienen un rol principalmente doméstico lo que supone una mayor exposición a éstos contaminantes (Simkovich et al, 2019) (Capistrano et al, 2017). Adicionalmente, surge una preocupación debida a la exposición a biomasa, la cual también afecta a población pediátrica que al estar bajo el cuidado de la madre se expone, si bien no en la misma medida, a gases y partículas nocivas producto de la combustión de biomasa traducidos en una mayor proporción de infecciones respiratorias agudas y asma comparados con población pediátrica no expuesta (Ramírez et al, 2019).
La relación entre la exposición a humo de biomasa y EPOC, entre otras enfermedades respiratorias, ha sido ampliamente descrita en la literatura médica a nivel mundial, donde se reportan RR de 3.2 (IC95\% 2.3-4.8) o 2.14 (IC95\% 1.78-2.58), según el estudio revisado, para mujeres mayores de 30 años que realizan tareas domésticas en áreas rurales (Capistrano et al, 2017), en revisión sistemática y meta análisis realizado se encontró un riesgo aumentado de EPOC asociado a combustión de biomasa en mujeres de 2.65 (IC95\% 2.13-3.31), así como se encontró mayor afectación en personas de área rural en comparación con las de área urbana dada la mayor disponibilidad de energía limpia en los últimos (Pathak et al, 2019).

Por otro lado, estudios sobre el fenotipo de la EPOC reportan un fenotipo específico inducido por humo de biomasa en el cual existe una obstrucción de flujo aéreo menos severa, mayor antracosis y mayores niveles sanguíneos de IgE reportados (Olloquequi et al, 2018) (Junemann y Legarreta, 2007), así como fenotipo específico para las mujeres, quienes, por características anatómicas propias del sexo presentan mayor engrosamiento bronquial traducido en mayor disnea y una disminución de FEV1 más vertiginosa (C. Gut-Gobert et al, 2019), este último de gran importancia dada la mayor exposición de las mismas a contaminantes derivados de la combustión de biomasa.

Sin embargo, esta relación no ha sido sólo de interés en el campo de la medicina pues su misma complejidad involucra otras disciplinas del conocimiento que desde su experticia han aportado para la comprensión, aunque aún incompleta, integral de esta problemática. Trabajos realizados han demostrado la presencia de Hidrocarburos Aromáticos Policíclicos (HAP) y material particulado como producto de combustión incompleta de material de biomasa, estos a su vez se reconocen como elementos potencialmente deletéreos para la salud respiratoria (Låg et al, 2020) (Gordon et al, 2014), a su vez, se investigan soluciones para disminuir la exposición de mujeres a estos contaminantes mediante el rediseño de estufas tradicionales de modo que estas sean más eficientes en cuanto a combustión del material de biomasa y eliminación de gases nocivos (Thomas et al., 2015) (Pope et al., 2021). 
Por parte de las ciencias sociales, particularmente de la antropología médica, se realizó un estudio sobre la población uruguaya, en el cual, se abordó la experiencia sensorial de la respiración especialmente en personas con EPOC quienes reportaban "falta de aire", encontrando de manera paradójica que el humo producto de la combustión de biomasa no es percibido como una amenaza que pueda empeorar los síntomas respiratorios dada su ubicuidad, de igual manera, se concluye que la EPOC "transforma el cuerpo y hace que sea hiperperceptivo e hipersensible a los cambios en el aire" (Wainwright, 2017).

La transdisciplinariedad es quizás un término complejo, sin embargo, puede ser definida de manera sucinta como "Un enfoque de investigación que tiene como objetivo fomentar la coproducción de conocimiento significativo a través de procesos integradores y participativos que reúnen a diversos actores, disciplinas y conocimientos bases, más allá de la cooperación de disciplinas (multidisciplinariedad) y la integración de disciplinas (interdisciplinariedad)" (Thompson et al, 2017) (ver tabla 2.), a su vez, el enfoque transdisciplinar introduce el concepto de "niveles de realidad", objeto de su estudio, que corresponde a las distintas percepciones de un sujeto, cada una de ellas invariable e incompleta en sí misma para la comprensión de la realidad, ello supone la transposición de estos niveles, rompiendo las reglas implícitas de cada uno para lograr una panorámica más amplia y veraz acerca de un tema en particular (Nicolescu, 2014).

Tal enfoque, durante los últimos años ha sido objeto de uso para diversos estudios, de los cuales la medicina no ha sido ajena, en donde fuera de generar nuevo conocimiento también se busca la resolución de problemas de la sociedad reconociendo su complejidad; de modo que exista un trabajo mancomunado que cierre la brecha entre científicos y actores sociales y se promueva el progreso de todas las partes involucradas (Ahedo García et al, 2020) (Paoli Bolio, 2020). Sin embargo, se reconoce que, por el carácter complejo de los temas que aborda, la transdisciplinariedad como enfoque es a su vez compleja en el sentido de unificación de conceptos y lenguaje que son propios de cada disciplina o actor participante, a su vez, las diferencias epistemológicas suponen dificultades en la comunicación entre disciplinas y por tanto este enfoque es demandante tanto en tiempo como en compromiso (Habermann et al, 2013).

Figura 2. Enfoques de investigación.

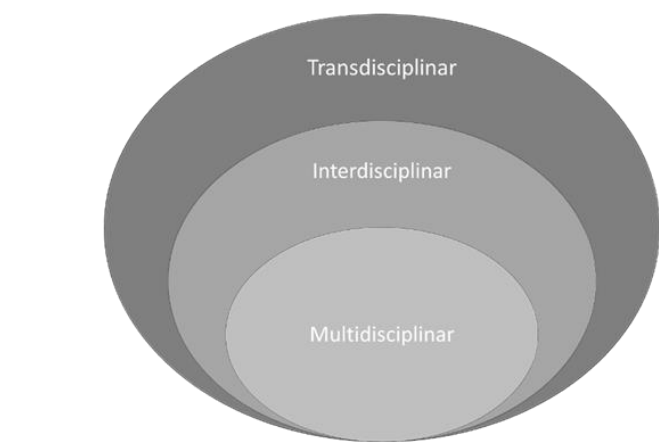

Fuente: Elaboración propia

Tabla 2. Diferencias entre multi-inter-transdisciplinariedad

\begin{tabular}{c|c|c}
\hline Multidisciplinariedad & Interdisciplinariedad & Transdisciplinariedad \\
\hline $\begin{array}{c}\text { No hay intercambio } \\
\text { de resultados }\end{array}$ & $\begin{array}{c}\text { Intercambio y } \\
\text { comparación de } \\
\text { resultados } \\
\text { Transferencia de } \\
\text { métodos y } \\
\text { un mistintas ópticas para }\end{array}$ & $\begin{array}{c}\text { Involucra a todos los } \\
\text { implicados en un } \\
\text { problema de } \\
\text { investigación }\end{array}$ \\
$\begin{array}{c}\text { Cada disciplina saca de los límites de las } \\
\text { sus propias } \\
\text { conclusiones }\end{array}$ & $\begin{array}{c}\text { Las disciplinas } \\
\text { permanecen } \\
\text { distinguibles }\end{array}$ & Niveles de realidad \\
\hline Fuente: Elaboración propia & \multicolumn{2}{l}{}
\end{tabular}

Un trabajo realizado que buscaba analizar la realidad socioeconómica y productiva de comunidades rurales del municipio de San Dionisio (Nicaragua) logró demostrar la necesidad de la transdisciplinariedad como metodología integradora que permitiera superar las dificultades de abordaje de la complejidad en el contexto rural representados por el "reduccionismo positivista de las ciencias naturales como hermenéutico de las ciencias sociales", y a su vez, generar estrategias de desarrollo para la región, destacando la participación ciudadana en su construcción (Alfaro-Mardones et al, 2015).

En el manual de métodos de investigación para la transformación rural del proyecto TRANSACT se nos presentan de manera pragmática, y basado en experiencias de trabajo realizadas en Etiopía, diferencias entre el trabajo inter y transdisciplinar 
enfocado hacia el desarrollo rural, donde si bien se resaltan las múltiples ventajas de la transdisciplinariedad, se deja evidencia de las diversas limitantes de esta metodología, las cuales, podrían considerarse dispendiosas al tratarse de un consenso entre las diferentes disciplinas y los actores sociales, de quienes han de tenerse en cuenta sus intereses particulares, miedos, reservas y formas de comunicación, aspectos que requieren de tiempo, dedicación y repetición de actividades (Habermann, 2013).

Ahora bien, la EPOC, dado su carácter multifactorial, sus relaciones pulmonares y extra pulmonares no lineares y su heterogeneidad entre quienes la padecen, aspectos representados en el impacto sobre la morbilidad y mortalidad de la población a nivel mundial, se posiciona como un problema social ampliamente complejo que requiere de estrategias multidimensionales para el perfilamiento de pacientes que permita, a su vez, el desarrollo de planes de manejo transdisciplinar que permitan un abordaje holístico e integral (Houben-Wilke, 2018), pese a ello, los estudios bajo el enfoque transdisciplinar de esta patología son escasos.

Programas como el Life Rainbow Programme (LRP) realizan intervenciones de este tipo en pacientes con enfermedades crónicas no neoplásicas, como la EPOC, reportando resultados positivos como mejora significativa de la calidad de vida y disminución la estancia hospitalaria (Law et al, 2020); por otra lado, el desarrollo de un programa de baile como medida de intervención a través de un enfoque transdisciplinar sugiere beneficios físicos y psicosociales en personas con sensación crónica de falta de aire que han de ser corroborados mediante un ensayo clínico aleatorizado (Harrison et al, 2020).

Sin embargo, en la actualidad no se tiene referencia de trabajos bajo esta modalidad que involucren tanto la EPOC secundaria a humo de biomasa como la ruralidad, lo cual, sin duda alguna, deja la puerta abierta para el desarrollo de investigaciones bajo esta metodología que permitan una visión más amplia de un problema que nos concierne a todos como sociedad.

\section{Conclusiones}

Los datos previamente expuestos dejan en evidencia la gran importancia que tiene la EPOC asociada a la cocción de alimentos mediante combustión de material de biomasa, en especial en mujeres de zonas rurales a nivel mundial. Está clara la relación entre ambos elementos, sin embargo, existen vacíos de orden multifactorial sobre cómo solucionar esta problemática. El enfoque de investigación transdisciplinar, pese al tiempo que requiere y la dificultad metodológica que representa, ofrece una alternativa válida y pertinente de abordaje de esta patología. La enfermedad cuenta con estudios realizados bajo esta metodología, sin embargo, resultan insuficientes para el contexto de una enfermedad con alta heterogeneidad, en la cual, los entornos rurales, donde hay mayor asociación al humo de biomasa, representan en sí mismos complejidad adicional que debe ser tenida en consideración.

\section{Bibliografía}

Agustí, A., \& Hogg, J. C. (2019). Update on the Pathogenesis of Chronic Obstructive Pulmonary Disease. New England Journal of Medicine, 381(13), 1248-1256.

doi:10.1056/nejmra1900475

Ahedo García, V. A. U., Álvarez, M., Arinyo i Prats, A., Barceló, J. A., Bocanegra Barbecho, L., Bogdánovic, I., ... \& Zurro, D. (2020). Terra Incognita: Libro blanco sobre transdisciplinariedad y nuevas formas de investigación en el Sistema Español de Ciencia y Tecnología. Pressbooks.

https://terraincognita.pressbooks.com/frontmatter/filiaciones/

Alfaro Mardones, J. I., Fernández Hernández, C. D. J., \& González García, M. D. J. (2015). La transdisciplinariedad una herramienta para apuntar al Buen Vivir. Polis. Revista Latinoamericana. $\quad 14 \quad$ (40), 23-42. http://dx.doi.org/10.4067/S071865682015000100002 .

Bălă, GP., Râjnoveanu, RM., Tudorache, E. et al. (2021). Air pollution exposure - the (in)visible risk factor for respiratory diseases. Environ Sci Pollut Res 28, 19615-19628 https://doi.org/10.1007/s11356-021-13208-X 
Banco Mundial (2018). Prevalence of current tobacco use (\% of adults). https://datos.bancomundial.org/indicator/SH.PR V.SMOK?end=2018\&most_recent_year_desc $=\mathrm{f}$ alse $\&$ start $=2018 \&$ view $=$ bar

Barnes, P. J., Burney, P. G. J., Silverman, E. K., Celli, B. R., Vestbo, J., Wedzicha, J. A., \& Wouters, E. F. M. (2015). Chronic obstructive pulmonary disease. Nature Reviews Disease Primers, 15076.

doi:10.1038/nrdp.2015.76

Capistrano, S. J., van Reyk, D., Chen, H., \& Oliver, B. G. (2017). Evidence of Biomass Smoke Exposure as a Causative Factor for the Development of COPD. Toxics, 5(4), 36. https://doi.org/10.3390/toxics5040036

Casanueva, E. (2008). Nutriología Médica, $3^{a}$. Edición. Editorial Medica Panamericana, 610-611

Celli, B. R., \& Wedzicha, J. A. (2019) Update on Clinical Aspects of Chronic Obstructive Pulmonary Disease. New England Journal of Medicine.; 381(13): 1257-1266.

doi:10.1056/nejmra1900500

Gordon, S. B., Bruce, N. G., Grigg, J., Hibberd, P. L., Kurmi, O. P., Lam, K. H., ... Martin, W. J. (2014). Respiratory risks from household air pollution in low and middle income countries. The Lancet Respiratory Medicine, 2(10), 823860.

doi:10.1016/s2213-2600(14)70168-7

Gut-Gobert C, Cavaillès A, Dixmier A, et al. (2019) Women and COPD: do we need more evidence? Eur Respir Rev; 28: 180055. doi: 10.1183/16000617.0055-2018.

Habermann, B., Misganaw, B., Peloschek, F., Dessalegn, Y., \& Yihenew, G. (2013). Inter-and Transdisciplinary Research Methods in Rural Transformation Case Studies in Northern Ethiopia. Centre for Development Research, University of Natural Resources and Life Sciences, BOKU, Vienna.
Harrison S, Bierski K, Burn N, et al. (2020) Dance for people with chronic breathlessness: a transdisciplinary approach to intervention development. BMJ Open Resp Res;7: e000696. doi:10.1136/bmjresp-2020-000696

Houben-Wilke S, Augustin IM, Vercoulen JH, et al. (2018) COPD stands for complex obstructive pulmonary disease. Eur Respir Rev.; 27: 180027 https://doi.org/10.1183/16000617.0027-2018

Juneman, Andrea, \& Legarreta, Gabriela (2007). Inhalación de humo de leña: una causa relevante pero poco reconocida de Enfermedad Pulmonar Obstructiva Crónica. Revista Americana de Medicina Respiratoria, 7(2),21-57. ISSN: 18521630.

https://www.redalyc.org/articulo.oa? $\mathrm{id}=3821383$ 70004

Låg, M., Øvrevik, J., Refsnes, M., \& Holme, J. A. (2020). Potential role of polycyclic aromatic hydrocarbons in air pollution-induced nonmalignant respiratory diseases. Respiratory Research, 21(1), 1-22. doi: 10.1186/s12931-020-01563-1.

Lopez, Matías, Mongilardi, Nicole, \& Checkley, William. (2014). Enfermedad pulmonar obstructiva crónica por exposición al humo de biomasa. Revista Peruana de Medicina Experimental y Salud Publica, 31(1), 94-99

Law M-C, Lau B H-P, Kwok AYY, Lee JSH, Lui RNY, Liu KH, Leung PPY, Chan CLW (2021). Empowering families facing endstage nonmalignant chronic diseases with a holistic, transdisciplinary, community-based intervention: 3 months' outcome of the Life Rainbow Program. Palliative and Supportive Care 19, 530-539. https://doi.org/10.1017/S1478951520001224

Ministerio de Salud y Protección social (2021). La importancia de prevenir la EPOC en pandemia. https://www.minsalud.gov.co/Paginas/Laimportancia-de-prevenir-la-EPOC-enpandemia.aspx.

Nicolescu, B. (2014). Multidisciplinarity, interdisciplinarity, indisciplinarity, and 
transdisciplinarity: Similarities and differences. RCC Perspectives, 2, 19-26.

http://www.jstor.org/stable/26241230

Olloquequi, J., Jaime, S., Parra, V., CornejoCórdova, E., Valdivia, G., Agustí, À., \& Silva O, R. (2018). Comparative analysis of COPD associated with tobacco smoking, biomass smoke exposure or both. Respiratory research, 19(1), 13.

https://doi.org/10.1186/s12931-018-0718-y

Paoli Bolio, Francisco José. (2019). Multi, inter y transdisciplinariedad. Problema anuario de filosofía y teoría del derecho, 13, 347-357. https://doi.org/10.22201/iij.24487937e.2019.13

Pathak, U., Naresh C, G., Jagdish C, S. (2019). Risk of COPD due to indoor air pollution from biomass cooking fuel: a systematic review and meta-analysis, International Journal of Environmental Health Research.

DOI: 10.1080/09603123.2019.1575951

Pope, D., Johnson, M., Fleeman, N., Jagoe, K., Duarte, R., Maden, M., ... \& Lewis, J. (2021). Are cleaner cooking solutions clean enough? A systematic review and meta-analysis of particulate and carbon monoxide concentrations and exposures. Environmental Research Letters, 16(8), 083002.

doi: 10.1088/1748-9326/ac13ec.

Ramírez-Venegas, A., Torres-Duque, C. A., Guzmán-Bouilloud, N. E., González-García, M., \& Sansores, R. H. (2019). Small airway disease in COPD associated to biomass exposure. Revista de Investigación Clínica, 71(1), 70-78.

Ramírez-Venegas, A., Velázquez-Uncal, M., PérezHernández, R., Guzmán-Bouilloud, N. E., Falfán-Valencia, R., Mayar-Maya, M. E., Aranda-Chávez, A., \& Sansores, R. H. (2018). Prevalence of COPD and respiratory symptoms associated with biomass smoke exposure in a suburban area. International journal of chronic obstructive pulmonary disease, 13, 1727-1734. doi: 10.2147/COPD.S156409

Rosário Filho, N. A., Urrutia-Pereira, M., D'Amato, G., Cecchi, L., Ansotegui, I. J., Galán, C. \&
Peden, D. B. (2021). Air pollution and indoor settings. World Allergy Organization Journal, 14(1), 100499.

https://doi.org/10.1016/j.waojou.2020.100499

Simkovich, S. M., Goodman, D., Roa, C., Crocker, M. E., Gianella, G. E., Kirenga, B. J., Wise, R. A., \& Checkley, W. (2019). The health and social implications of household air pollution and respiratory diseases. NPJ primary care respiratory medicine, 29(1), 12.

doi: 10.1038/s41533-019-0126-x

Thomas, E., Wickramasinghe, K., Mendis, S., Roberts, N., \& Foster, C. (2015). Improved stove interventions to reduce household air pollution in low and middle income countries: a descriptive systematic review. BMC public health, 15(1), 115.

doi: 10.1186/s12889-015-2024-7.

Thompson, M. A., Owen, S., Lindsay, J. M., Leonard, G. S., \& Cronin, S. J. (2017). Scientist and stakeholder perspectives of transdisciplinary research: Early attitudes, expectations, and tensions. Environmental Science \& Policy, 74, 30-39.

doi:10.1016/j.envsci.2017.04.006

Wainwright, M. (2017) Sensing the Airs: The Cultural Context for Breathing and Breathlessness in Uruguay, Medical Anthropology, 36:4, 332-347, DOI: $10.1080 / 01459740.2017 .1287180$

World Health Organization (2021). Tabaco. https://www.who.int/es/news-room/factsheets/detail/tobacco

World Health Organization. (2006). Air quality guidelines: global update 2005: particulate matter, ozone, nitrogen dioxide, and sulfur dioxide. World Health Organization.

Zambrano Bohórquez, D. L. (2017). Concentración de material particulado por combustión de leña en la zona rural del municipio de Santa Sofía, Boyacá. Universidad Militar Nueva Granada. 